\title{
Reviewing the Recent Developments in Idiopathic Intracranial Hypertension
}

\author{
Jasvir Virdee (D) - Stephanie Larcombe (D) - Vivek Vijay • \\ Alex J. Sinclair (D) - Margaret Dayan · Susan P. Mollan (D)
}

Received: February 19, 2019 / Published online: September 9, 2020

(C) The Author(s) 2020

\begin{abstract}
There is increasing evidence and appreciation of idiopathic intracranial hypertension (IIH) in medicine. The pathological processes underlying raised intracranial pressure are being studied, with new insights found in both hormonal dysregulation and the metabolic neuroendocrine axis. These will potentially lead to novel therapeutic targets for IIH. The first consensus
\end{abstract}

Digital Features To view digital features for this article go to https://doi.org/10.6084/m9.figshare.12547667

Jasvir Virdee and Stephanie Larcombe are joint first authors.

J. Virdee

Ophthalmology, Queen Elizabeth Hospital, University Hospitals Birmingham NHS Foundation

Trust, Birmingham, UK

S. Larcombe

Medical School, University of Birmingham,

Birmingham, UK

V. Vijay · A. J. Sinclair

Metabolic Neurology, Institute of Metabolism and

Systems Research, University of Birmingham,

Birmingham, UK guidelines have been published on the investigation and management of adult IIH, and the International Headache Society criteria for headache attributable to IIH have been modified to reflect our evolving understanding of IIH. Randomized clinical trials have been published, and a number of studies in this disease area are ongoing.

Keywords: Headache; Idiopathic intracranial hypertension; Lumbar puncture; Obesity; Papilloedema; Pseudotumour cerebri; Raised ICP; Weight management

V. Vijay · A. J. Sinclair

Centre for Endocrinology, Diabetes and Metabolism, Birmingham Health Partners, Birmingham, UK
A. J. Sinclair · S. P. Mollan ( $ه)$
Birmingham Neuro-Ophthalmology, Queen
Elizabeth Hospital, University Hospitals
Birmingham NHS Foundation Trust, Birmingham, UK
e-mail: susan.mollan@uhb.nhs.uk
M. Dayan
Newcastle Eye Centre, The Newcastle Upon Tyne Hospitals NHS Foundation Trust, Royal Victoria Infirmary, Newcastle upon Tyne, UK 


\section{Key Summary Points}

\section{Why carry out this study?}

Investigation and management strategies have been recommended by the first consensus guidance for IIH.

Understanding the underlying pathophysiology of IIH is identifying novel avenues for targeted therapies.

What was learned from the study?

This review summarizes the IIH pathophysiology known to date.

It presents an evaluation of the randomized controlled trials in IIH.

It details the potential targets for future disease management.

\section{DIGITAL FEATURES}

This article is published with digital features to facilitate understanding of the article. To view digital features for this article go to https://doi. org/10.6084/m9.figshare.12547667.

\section{INTRODUCTION}

Idiopathic intracranial hypertension (IIH), also known as primary pseudotumour cerebri syndrome, is a neurological disorder characterised by raised intracranial pressure (ICP) that can cause papilloedema with no identifiable cause found $[1,2]$. There is a rising incidence of this disease, which is most common in females of working age $[3,4]$, and appears to be related to the prevalence of obesity $[4,5]$, the major known risk factor for the disease $[1,6]$. IIH is associated with headache, which impacts quality of life [7]; over half of those with IIH report ongoing headaches at 12 months following initial diagnosis [8]. IIH can cause visual loss, even to the level of severe visual impairment or legal blindness [9]. Current medical treatments are often poorly tolerated [10], and the overarching research aspirations of both patients and clinicians are to understand the aetiology and management of IIH, with particular focus on headache [11]. The recent guidance in IIH acknowledges uncertainties in many aspects of the disease [1]. Novel targets are now being identified, and insights into monitoring disease activity are improving [12]. This article is based on previously conducted studies and does not contain any studies with human participants or animals performed by any of the authors.

\section{EPIDEMIOLOGY OF IIH}

IIH is considered a rare disease; however, increasing numbers of patients are being reported. Previously, the incidence in the general population was reported to be between 0.5 and 1.0 per 100,000 [5]. Comparing rates within the United Kingdom (UK) between the years 2007 and 2008, Raoof et al. found the incidence of IIH was 1.56 per 100,000 for men and 2.86 per 100,000 for women [12, 13]. Recent evidence indicates a greater than $100 \%$ increase in the incidence of IIH in England between 2002 and 2016, which rose from 2.3 to 4.7 per 100,000 in the general population [3]. In females, the incidence of IIH more than tripled between 2005 and 2017, from 2.5 to 9.3 per 100,000 person-years, and increased markedly in those with a body mass index (BMI) higher than $30 \mathrm{~kg} / \mathrm{m}^{2}$ [4]. Adult women are more likely to develop IIH than men, with a peak incidence in females aged 25 years of 15.2 per 100,000 [3]. Worldwide, IIH is more common in countries such as the UK [3, 13], Italy [14], Israel [15] and the USA [16], while incidence is lower in Asia $[17,18]$. A recent systematic review identified a positive correlation between a country-specific prevalence of obesity and incidence of IIH [6].

The incidence of IIH in childhood is estimated to be around 0.5 per $100,000[19,20]$. Paediatric IIH has been classified by anthropometric features, with three identifiable subgroups of paediatric IIH: a young cohort that is not overweight, an early adolescent group that is either overweight or obese, and a late 
adolescent group that is mostly obese. This indicates that the underlying pathological mechanisms for IIH may differ depending on age and BMI [21].

\section{PATHOPHYSIOLOGY}

The pathological processes underlying elevated ICP in IIH patients are unknown. Current research is focused on understanding the respective roles of obesity, increased cerebrospinal fluid (CSF) production and hormonal dysregulation. Additional theoretical mechanisms, such as venous hypertension and reduced CSF absorption, may also play a role in the pathophysiology of $\mathrm{IIH}[2,22,23]$. There is however an emerging theme that IIH is a neurometabolic disease [23]. Adderley et al. found a twofold increase in cardiovascular disease risk in women with IIH compared to age-, sex- and, most importantly, BMI-matched controls [4]. This suggests that multiple factors may be driving the disease.

\section{Obesity}

There is a well-established link between IIH and obesity $[6,24]$; the prevalence of IIH in populations with a higher BMI is greater than in the general population [5]. The dramatic rise in obesity rates globally [25] is mirrored in the rising incidence of $\mathrm{IIH}[3,5,26]$. Daniels et al. found that, compared to controls, patients with $\mathrm{IIH}$ reported higher weight gain in the 1-year period prior to the onset of the disease. Higher BMI and weight gain percentage were also associated with higher (dose-dependent) risk of IIH. Interestingly, weight gain of just $5-15 \%$ can lead to an increased risk of IIH [27]. Ko et al. found that patients who experienced a recurrence of IIH following symptom resolution showed weight gain of just $6 \%$ of total body weight [24]. Records were analysed over a 16-year period at two hospitals, and the authors found that 26 out of the 50 patients analysed had IIH recurrence (increase in an average BMI of 1.3 per year), and the 24 who had no recurrence did not gain weight (average decrease in BMI of 0.96 per year) [24]. This shows the exquisite sensitivity between even modest weight gain and recurrence of IIH. Adderley et al. reported that the incidence of IIH increased rapidly above a BMI of $30 \mathrm{~kg} / \mathrm{m}^{2}$ [4]. This evidence reinforces the close link between obesity and IIH.

Lifestyle interventions such as diet and exercise have long been known to be an effective treatment for $\mathrm{IIH}$; the first study to demonstrate the effectiveness of a lifestyle intervention for improvement of IIH symptoms was carried out in 1974 [28]. In the mid-1990s, the first cases of bariatric surgery for IIH were reported [29]. Previous literature shows that between 3 and 15\% loss in body weight is required to put the disease into remission. An objective evaluation of weight loss in IIH was performed as a prospective crossover cohort trial using a very low-calorie meal replacement to induce significant weight loss, defined as a loss of $15 \%$ or greater of total body weight. Such weight loss resulted in significantly lowered ICP and improved papilloedema, visual function (perimetry and acuity) and headache outcomes (50\% improvement in headache frequency, severity and frequency of analgesic use) [30]. In the Idiopathic Intracranial Hypertension Treatment Trial (IIHTT), a randomised controlled trial (RCT) investigating the use of acetazolamide in IIH patients with mild visual loss, the treatment group used acetazolamide, and both groups (placebo and treatment) were provided a low-sodium diet with behavioural weight management support. The treatment group experienced an average $6 \%$ weight loss compared to $3 \%$ weight loss in the controls [31]. The IIHTT used a psychological statistical method to report that weight loss did not mediate the treatment effect of acetazolamide.

Distribution of body fat in IIH has been investigated, and it was demonstrated that truncal fat mass was the only parameter that correlated with lumbar puncture pressure, as compared with limb fat mass, BMI, waist circumference and total fat mass [32]. A relationship has been established between truncal weight loss in patients with IIH and a reduction in disease activity [30].

The relationship between obesity and IIH in men is less clear, although men appear to be at 
risk of greater vision loss and obstructive sleep apnoea if they have IIH [33].

\section{CSF Dysregulation}

CSF is secreted by the choroid plexus, which consists of epithelial cells lining the ventricles in the brain. CSF secretion is driven by the net movement of sodium ions $\left(\mathrm{Na}^{+}\right)$from the blood to the cerebral ventricles, creating an osmotic gradient down which water moves. Although several channels are involved in this process, the principle channel and rate-limiting step is the $\mathrm{Na}^{+}$- and $\mathrm{K}^{+}$-dependent adenosine triphosphatase $\left(\mathrm{Na}^{+} / \mathrm{K}^{+} /\right.$ATPase $)$that actively transports $\mathrm{Na}^{+}$into the cerebral ventricle $[34,35]$. CSF flows through the ventricles and into the subarachnoid space, before being returned to the bloodstream via arachnoid granulations.

Specific inhibition of $\mathrm{Na}^{+} / \mathrm{K}^{+} /$ATPase with ouabain has been shown to reduce CSF secretion by $70-80 \%$ [36].

Aquaporins (AQPs), membrane-bound water transport channels, are thought to be involved in the osmotic homeostasis of the brain. Research has not demonstrated a pathogenic role for AQP4 in the genesis of IIH [37]. AQP1, another channel, appears to be important in drug-induced elevation of ICP, and animal studies show a link between obesity, AQP1 expression and raised ICP [38]. However, no investigations of the role of AQP1 in IIH in humans have yet been reported.

Impaired drainage of CSF into the subarachnoid space is another potential source of IIH. The turnover of CSF was found to be reduced in patients with papilloedema [39]. Patients with IIH have been shown to have abnormalities in blood clotting [40], and it has been suggested that microthrombi could cause impaired CSF drainage in IIH [41]. However, no direct evidence of microthrombi causing raised ICP has been shown to date.

\section{Glucagon-Like Peptide 1}

Glucagon-like peptide-1 (GLP-1) is an incretin with weight-modifying properties, and has been shown to have a natriuretic effect in the kidney through inhibition of the $\mathrm{Na}^{+} / \mathrm{H}^{+}$exchanger in proximal tubule cells. CSF secretion, as discussed above, is controlled by $\mathrm{Na}^{+} / \mathrm{K}^{+} /$ATPase channels and pumps fluid into the ventricles akin to an inverted renal proximal tubule. Exploratory work showed that the choroid plexus expresses GLP-1 receptor [42]. Botfield et al. thus hypothesised that GLP-1 could modulate CSF secretion at the choroid plexus, thereby mediating ICP [42]. Following from this, it was demonstrated that exendin-4, a GLP1 receptor agonist, significantly reduced the activity of $\mathrm{Na}^{+} / \mathrm{K}^{+} /$ATPase. In a rodent study, Botfield et al. showed that exendin- 4 led to a $65 \%$ reduction in ICP within $30 \mathrm{~min}$ of administration, and that its effect on ICP was cumulative. This finding is now being translated into the IIH Pressure Clinical Study (IIHPCS; ISRCTN12678718). The IIH pressure study is a double-blinded, placebo-controlled physiology study assessing the effects of exenatide on ICP in patients with active IIH, and is expected to report soon.

\section{Glucocorticoid Metabolism}

Glucocorticoid metabolism has previously been characterised in IIH subjects before and after therapeutic weight reduction. 11 $\beta$-Hydroxysteroid dehydrogenase type 1 (11 $\beta$-HSD 1$)$ is an intracellular enzyme that converts inactive cortisone into the active cortisol. This amplifies local glucocorticoid activity independent of systemic cortisol [43]. Global 11 $\beta$-HSD1 activity was found to decrease with weight loss, and a significant correlation was found between the reduction in ICP and the decrease in global $11 \beta$ HSD1 activity [44]. It is now known that $11 \beta$ HSD1 is expressed and has activity in the choroid plexus epithelial cells, along with other key elements of the glucocorticoid signalling pathway. Inhibitors of $11 \beta-H S D 1$, such as oral AZD4017, have been developed for type 2 diabetes mellitus, obesity and metabolic syndrome. The IIH Drug Trial hypothesised that specific inhibition of $11 \beta$-HSD1 using such drugs may decrease ICP in individuals with IIH, which could open an entirely novel therapeutic 
avenue for targeting the disease [45]. The results of this trial are presented later in this article.

\section{Hormonal Dysregulation}

Hormonal dysregulation has been shown to play an important role in the pathogenesis of IIH $[2,22,23]$. Androgens have been identified as potential key players in IIH pathophysiology. Serum testosterone, and the enzyme $5 \alpha$-reductase involved in its metabolism, has been shown to be higher in those with IIH compared to ageand BMI-matched controls [46]. In a recent study that comprehensively compared the systemic and CSF androgen metabolome in women with IIH to sex-, BMI- and age-matched control groups with either simple obesity or polycystic ovary syndrome (PCOS), IIH women showed a pattern of androgen excess with increased serum testosterone and increased CSF testosterone and androstenedione [47]. This was found to be distinct from that observed in PCOS and simple obesity. The authors went on to show that human choroid plexus expressed the androgen receptor, alongside the androgen-activating enzyme aldo-keto reductase type 1C3, and in a rodent model testosterone significantly enhanced the activity of $\mathrm{Na}^{+} / \mathrm{K}^{+}$ATPase, a surrogate of CSF secretion. In summary, androgens can modulate CSF secretion via the choroid plexus, and these findings implicate androgen excess as a potential causal driver for IIH and therefore a potential therapeutic target [47].

The hormone leptin plays an important role in the regulation of fat storage. Leptin is secreted by adipocytes, and its concentration in the body correlates with total body fat [48]. Higher serum leptin has been demonstrated in IIH patients, controlling for age and BMI, indicating that leptin dysregulation may be important in IIH [49]. Another study showed that leptin was elevated in the CSF of IIH patients, though there was no correlation with leptin CSF levels and BMI [50]. However, leptin is secreted disproportionately from subcutaneous fat compared to visceral fat [51], and since neither of these studies accounted for fat distribution, elevation of leptin in IIH patients may be a secondary effect of non-central body adiposity.
These studies indicate that the role of leptin in IIH warrants further investigation.

\section{Venous Hypertension}

Increased dural venous sinus pressure has been proposed as a potential mechanism underlying IIH. Neuroimaging studies have established that venous sinus stenosis is a common finding among patients with IIH $[52,53]$, yet the extent of stenosis and the clinical course of IIH are still to be fully correlated [52]. Reducing the ICP by removal or diversion of CSF has been shown to reduce dural venous stenosis [54], and these findings may indicate that the stenosis may be a consequence, rather than a cause, of IIH [55]. Despite good evidence of the effect of stenting on the venous sinus pressure gradient, the effect on CSF pressure is less clearly understood. There are many uncontrolled institutional case-based series reporting resolution of papilloedema and improvement in headache following venous sinus stenting, which is discussed below in management of IIH.

\section{CLINICAL PRESENTATION}

Headache is present in over 90\% of people diagnosed with IIH. The phenotype of increased ICP headache is no longer thought of as an early morning headache [56], and migraine is now reported to be the predominant phenotype [57]. The International Headache Society criteria have been modified to reflect that improvement in headache with ICP reduction is no longer a requirement as a diagnostic criterion of headache attributable to IIH [58]. Pulsatile tinnitus, either unilateral or bilateral, is commonly reported in IIH. Transient visual obscurations are common in IIH and are described by patients as 'greying' or 'blacking out' of vision in one or both eyes and lasting seconds. Transient visual obscurations are usually associated with changes in posture. Unilateral or bilateral sixth-nerve palsy may occur secondary to raised ICP and cause horizontal diplopia [22]. Cognitive function has been reported to be affected in IIH [59]. 
To confirm a diagnosis of IIH, papilloedema needs to be present, and care must be taken in the examination of the optic nerve, as papilloedema can be misdiagnosed. This has led to unnecessary investigations, procedures and therapy, with the leading reason being the incorrect interpretation of the optic nerve appearance during clinical examination [60]. Examination of the eye can be challenging, and where diagnostic uncertainty exists, the guidelines recommend that papilloedema be confirmed by an experienced specialist $[1,61]$.

The detection of papilloedema by fundus photography is extremely sensitive, even among non-specialists such as emergency medicine doctors. However, it is of note that none of the cases presented in a recent study by Blanch et al. had subtle papilloedema (Frisén grade 1), which can present the diagnostic dilemma of papilloedema versus pseudopapilloedema [62]. Other imaging tools such as optical coherence tomography (OCT) and fundus fluorescence angiography (FFA) are helpful $[1,61]$. More evidence of the utility of OCT and OCT angiography in defining papilloedema from pseudopapilloedema is emerging [63, 64]. These findings need to be validated across the different OCT device platforms and larger data sets to enable clinicians to implement their findings in routine clinical care.

\section{INVESTIGATION OF PAPILLOEDEMA}

In 2018, the first consensus IIH guidance was published [1]. The IIH guidelines highlighted the importance of accurate clinical phenotyping, particularly when diagnosing papilloedema $[1,65]$. On confirmation of true papilloedema, comprehensive parameters of visual function, including visual acuity, colour vision, pupil assessment, dilated fundus examination and formal visual fields, should be noted, as this will determine the management course. Blood pressure must be measured to exclude malignant hypertension. Neuroimaging should then be performed to exclude secondary causes of elevated ICP and to identify structural alterations associated with raised ICP. Typical neuroimaging features of raised ICP include a partial empty sella turcica, flattening of the posterior optic globe, enlargement of the optic nerve sheath and increased tortuosity of the optic nerve. CT or MR venography should be performed to exclude a venous sinus thrombosis, which may mimic IIH in up to a third of cases [66]. As there is different treatment for venous sinus thrombosis, and delay in the diagnosis potentially causes significant morbidity, it is recommended that venography be performed at the same time as initial neuroimaging $[1,61]$.

Lumbar puncture (LP) is mandatory and forms part of the diagnostic criteria for IIH [67] in addition to normal CSF composition analysis. Diagnostic criteria require that opening pressure be measured in the lateral decubitus position with stretched legs, and without sedative medications. There is increasing consensus that there is no single absolute cut-off which is abnormal, but there is indication of a 'grey zone' at which the LP opening pressure may become increasingly pathological (between 25 and $30 \mathrm{~cm} \mathrm{CSF)} \mathrm{[1,} \mathrm{61].}$

Blood tests may be performed to investigate for secondary causes of raised ICP. Other medical conditions such as anaemia, endocrine causes and vitamin A excess may present with symptoms similar to IIH $[68,69]$. In those patients who do not fit the typical phenotype for IIH (i.e. female, of childbearing age and who have a BMI greater than $30 \mathrm{~kg} / \mathrm{m}^{2}$ ), a thorough drug history and exclusion of endocrine causes is required to exclude an underlying cause $[1,61]$.

\section{MANAGEMENT OF IIH}

The guidelines set out three key principles for the management of IIH: first, addressing the underlying modifiable risk factor of weight gain; second, protecting the vision through regular assessment and escalation of treatment when sight is threatened; and finally, reducing headache morbidity through active management [1]. The specialist interest group involved in establishing these guidelines recognised that a small proportion of people with IIH are at risk of rapid permanent visual loss [9] and require 
urgent surgical management, termed fulminant IIH (Table 1) [1]. The guideline touched on finer areas of practice including co-morbid conditions, pregnancy, managing headache in the shunted patient and IIH without papilloedema. It also gave consensus recommendations for the timely follow-up of patients reflecting the status of their optic nerve head and visual field assessment [1]. The major achievement of this document is the interdisciplinary effort to provide a framework for equal care for those with IIH, particularly within the UK. Many aspects are applicable in other countries, as supported by the subsequent publication of the first European Headache Federation Guidelines for IIH [61].

\section{Management of Fulminant IIH}

Fulminant IIH is defined as IIH resulting in a significant reduction in visual function within 4 weeks of diagnosis [1]. Those with fulminant IIH require emergency surgical management, aimed at rapidly lowering the CSF pressure [70]. Should surgery be delayed, a lumbar drain can be inserted to protect vision in the intervening period [1]. A large systematic review of 109 studies over a 34-year period showed that CSF diversion procedures for IIH reduced papilloedema in $78.9 \%$ of cases and prevented visual field deterioration in $66.8 \%$ [71]. The disadvantages of shunts are that they are invasive, carry an infection risk and may require revisions in up to one-third of patients [72]. Ventriculoperitoneal shunts are preferred due to the reported lower revision rates compared with lumbo-peritoneal shunts (1.8 versus 4.3 revisions per patient, respectively) [73]. Adjustable valves (antigravity or anti-siphon devices) can reduce the risk of low-pressure headaches in the long term [73]. However, it is important to counsel patients that ventriculoperitoneal shunt insertion leads to a temporary driving restriction in some countries, such as the UK [1]. Optic nerve sheath fenestration (ONSF) surgery is another option. Disadvantages of this surgical approach include persistently raised ICP and the severe complication of risk of vision loss, as well as other complications such as diplopia [74].

\section{Managing Weight Loss}

Weight loss has been shown to be an effective method for improving headache symptoms, papilloedema and reducing ICP [30]. Sustaining weight loss is difficult, with patients on average regaining one-third to one-half of the weight that was lost at 1 year and returning to their original weight within 5 years [75]. For IIH, a weight gain of $6 \%$ has led to documented recurrences [24].

Bariatric surgery is the most effective method for sustained weight loss, resulting in $15-30 \%$ weight loss over 15-20 years depending on the surgical procedure [76]. Gastric bypass (Rouxen-Y) surgery has been shown to be an effective method for reducing weight for IIH and can result in reduction or resolution of papilloedema, headache and tinnitus, with a concomitant reduction in ICP [29]. In addition, there is a decrease in circulating leptin, glucose, insulin and IGF-1, and an increase in adrenocorticotropic hormone (ACTH), following bypass surgery [77]. Gastric sleeve surgery has also been reported to be useful in IIH [78], leading to sufficient weight loss and resolution of papilloedema. Bariatric surgery is currently being investigated in the Idiopathic Intracranial Hypertension Weight Trial (IIH:WT), an RCT assessing bariatric surgery against a community weight-loss programme [79].

\section{Pharmacological Therapy in IIH}

Acetazolamide, a carbonic anhydrase inhibitor, is the main pharmacological therapy for IIH. Although class I evidence exists for its use in IIH, only two RCTs to date have examined its efficacy [10, 31]. In 2014, a large randomised, double-blind placebo-controlled study of acetazolamide combined with a low-sodium diet was published [31]. The IIH treatment trial (IIHTT) showed that the mean decrease in perimetric mean deviation (PMD) was statistically significant for those with mild visual loss using a low-calorie diet plus acetazolamide, 
compared with a low-calorie diet and placebo, at 6-months follow-up. In the IIHTT, the benefits were most marked in those with the most significant papilloedema. High doses of acetazolamide were used, with more than $40 \%$ of patients treated with $4000 \mathrm{mg}$ of acetazolamide daily. This dose may not be tolerable in a realworld setting, as previous studies have demonstrated that $48 \%$ of patients discontinue acetazolamide at daily doses of $1500 \mathrm{mg}$ [10]. Side effects of acetazolamide include paraesthesia, dysgeusia, vomiting and diarrhoea, as well as malaise, fatigue and depression [1, 10]. A 2015 Cochrane review [80] highlighted that the available data are not of sufficient quality, and therefore the evidence for the use of acetazolamide in IIH is currently limited. Hence, not all clinicians routinely prescribe acetazolamide for IIH [1].

Topiramate is a migraine prophylactic with mild carbonic anhydrase inhibitor activity and additional appetite suppressant action. Topiramate has been reported in an open-label study comparing its efficacy against acetazolamide [81]. The dual action of reducing CSF secretion and reducing migraine is appealing to clinicians. A recent in vivo animal study investigated clinical (single equivalent human dose) and high (daily equivalent human dose) subcutaneous administration of many of the known drugs used in IIH. Topiramate lowered ICP in female rats by $21 \%$ over peak plasma drug concentration at $2 \mathrm{~h}(p=0.015)$ and $32 \%$ $(p=0.0009)$, respectively. Of note, there was no significant reduction in ICP noted with human equivalent doses of acetazolamide $4000 \mathrm{~g}$, amiloride $20 \mathrm{mg}$, octreotide $2 \mathrm{mg}$ or furosemide $240 \mathrm{mg}$. The report also examined oral administration of topiramate and found that it significantly lowered ICP by $22 \% \quad(p=0.018)$, compared to a 5\% reduction with acetazolamide ( $p=>0.999$ ) [82]. Human physiological studies would be further informative in assessing ICP levels in response to common medications used. Topiramate has also been used successfully to treat headache in IIH. Common side effects include reduced appetite, paraesthesia, and cognitive and concentration impairment. Caution is advised for those with prior history of depression, and due to its teratogenicity, appropriate contraception is recommended in women who are of reproductive age $[1,61]$.

11ß-hydroxysteroid dehydrogenase type 1 (11 $\beta$-HSD1) blocking agents have recently been identified as potentially beneficial in IIH. The choroid plexus expresses 11ß-HSD1 to amplify cortisol availability and also aids in the regulation of CSF production [43]. The IIH Drug Trial was a recent RCT comparing the selective $11 \beta$ HSD1 inhibitor AZD4017 versus placebo. Thirty-one patients were randomised to either the intervention $(n=17)$ or control $(n=14)$. This trial highlighted that AZD4017 was safe, well tolerated and showed trends towards ICP reduction [83]. An exploratory analysis assessing mean change in LP pressure within each group found a significant decrease in the AZD4017 group [mean change: $-4.3 \mathrm{cmH}_{2} \mathrm{O}$ $(\mathrm{SD}=5.7) ; P=0.009$ ] but not in the placebo group [mean change: $-0.3 \quad \mathrm{cmH}_{2} \mathrm{O}$ $(\mathrm{SD}=5.9) ; P=0.8$ ] [83]. The authors therefore highlight that this drug may be useful in treating IIH, with improved tolerability compared to the currently used medications.

\section{Other Interventions for IIH}

Anecdotally, patients have reported that diagnostic lumbar puncture (LP) improves headache in IIH. A cohort study demonstrated that LP does indeed improve headache symptoms, but only to a mild degree, and with variable success across studies [84]. Post-LP exacerbation headache is common, particularly in patients reporting mild headache on the day of the procedure, where $81 \%$ reported such an exacerbation [84]. Furthermore, $40 \%$ of patients report 'severe pain' and $47 \%$ of patients report 'extreme anxiety' associated with the procedure [85]. Therefore, consensus guidelines do not support the use of serial LP without discerning evidence [86]. The increasing use of telemetric ICP monitors will provide valuable insights into CSF dynamics and the effects of therapeutic LP in the future [12]. Dural venous sinus stenting (VSS) is an evolving area of research for those identified with a focal venous sinus stenosis, as discussed in the pathogenesis above [87-89]. A 

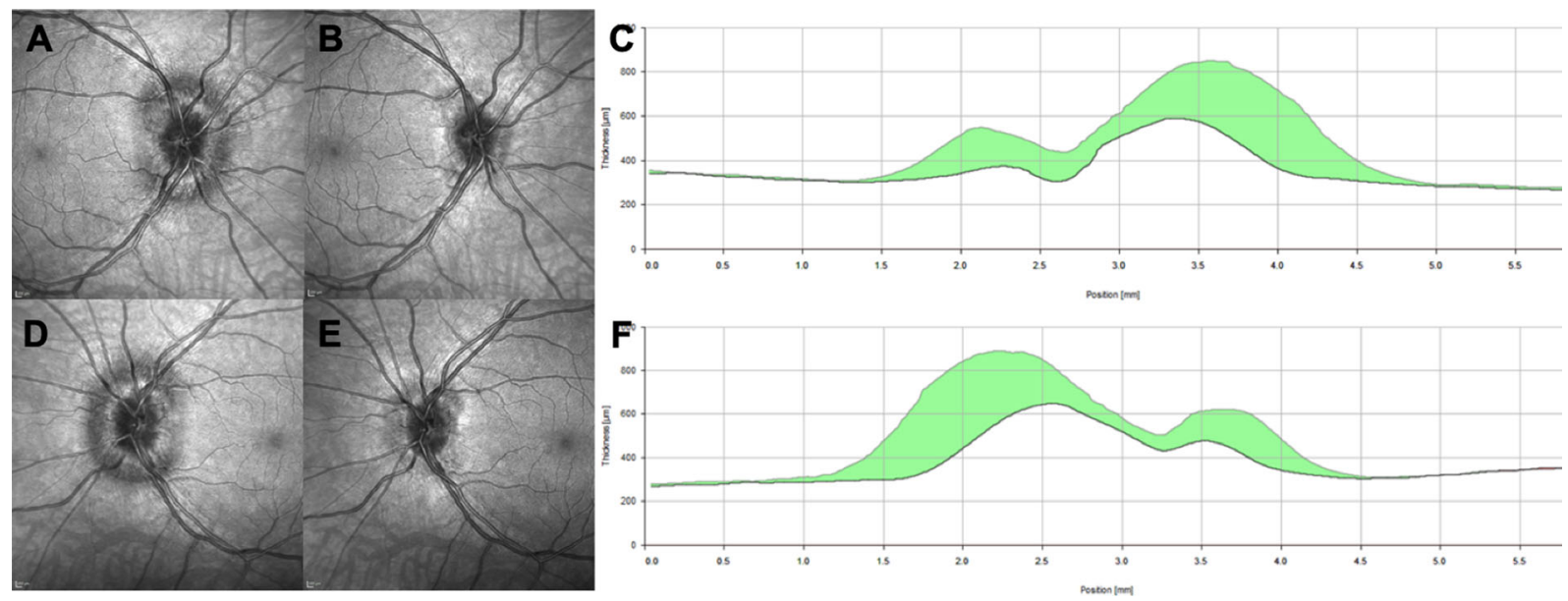

Fig. 1 Optical coherence tomography (OCT) images showing change in disc swelling over the time period. a Infrared image of the right optic nerve at presentation; b infrared image of the right optic nerve following weight loss; c the central cross section and the amount of reduction in the retinal nerve fibre layer in the right eye.

recent meta-analysis of 474 patients confirmed papilloedema resolution in $94 \%$ and improved headache outcomes in $77 \%$ of cases [90]. The most common side effect in up to half of those treated is transient headache of less than 7 days, usually ipsilateral to the stent [91]. Rarer, but serious, side effects that have been reported include intracranial haemorrhage, venous sinus thrombosis, femoral pseudoaneurysm, transient hearing loss, allergic reaction to contrast, retroperitoneal hematoma, neck hematoma, stent migration and death. In the meta-analysis, complications occurred in $2 \%$ of patients, while recurrence of IIH symptoms following VSS occurred in $10 \%$ of cases [90]. All advocate anticoagulation, with the majority being heparinised prior to the procedure, with subsequent maintenance therapy for a period of 6 months up to life. Overall, the results which have been published thus far are encouraging, although they are drawn from observational case series and are thus susceptible to a number of biases. A recent review by Gurney et al. highlighted the need for robust RCTs to investigate venous sinus stenting in the management of IIH [92]. d Infrared image of the left optic nerve at presentation; e infrared image of the left optic nerve following weight loss; $\mathbf{f}$ the central cross section and the amount of reduction in the retinal nerve fibre layer in the left eye

\section{PATIENT MONITORING AND PROGNOSIS}

The course of disease in IIH is variable. Visual monitoring is essential for patients with active disease, and where there is deteriorating visual function, a step in management may be required. Imaging the fundus (e.g. colour fundus photography) is useful for longitudinal assessment. Optical coherence tomography (OCT) is increasingly being utilised to objectively monitor changes in the retinal nerve fibre layer (RNFL) and the optic nerve head disc $(\mathrm{ONH})$ volume to track papilloedema. For example, Figs. 1 and 2 show the improvement in the RNFL and ONH volume, respectively, in a 29-year-old female Caucasian who was diagnosed with IIH. Through lifestyle and behavioural modification alone, she lost $17 \mathrm{~kg}$ over 6 months. Her BMI reduced from 35.6 to $28.8 \mathrm{~kg} / \mathrm{m}^{2}$. OCT RNFL must be interpreted cautiously, as a reduction in elevation of the RNFL may indicate resolving papilloedema and/ or loss of retinal nerve fibres leading to optic atrophy. Therefore, the use of macular ganglion cell analysis may be more helpful in IIH [93].When imaging papilloedema, the scans can 

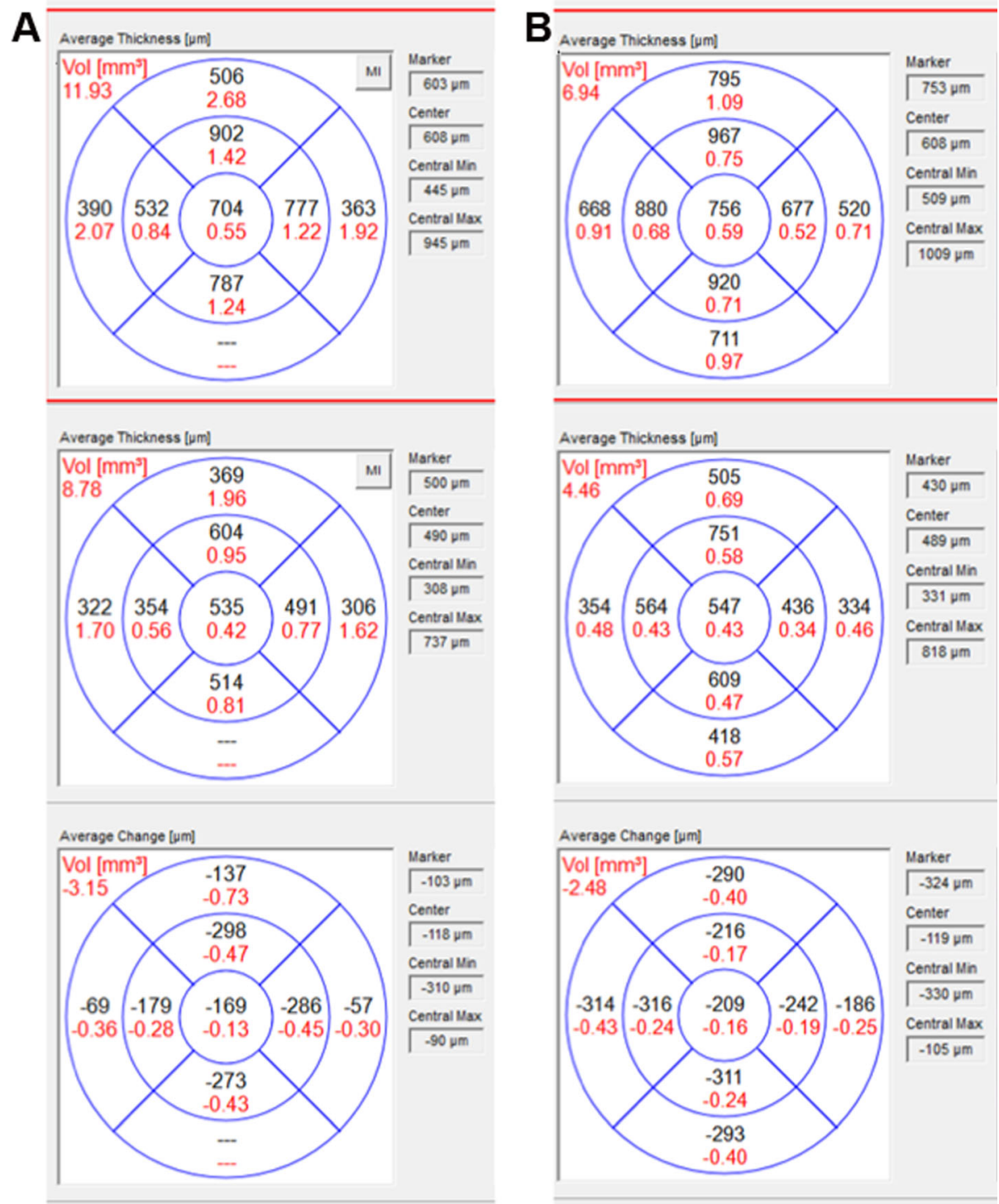

Fig. 2 The disc volume segments and change in volume over the time period. Top image-latest, middle image-at presentation, bottom image-change in volume. a Right eye. $\mathbf{b}$ Left eye

be prone to proprietary software segmentation error; therefore a qualitative assessment of the traces within the OCT should be made and adjustments in the segmentation made to ensure the reliability of the OCT parameters for longitudinal follow-up [94].

The severity of papilloedema, as graded using the modified Frisén scale, has been shown to be a predictor of final visual outcome, rather than cotton wool spots or retinal hemorrhages as previously thought [95]. Racial disparities may exist, with poorer visual outcomes in those of
African ancestry compared with White Americans [96]. Other studies have examined the anatomical changes in the optic canal to predict visual outcome, but these have shown conflicting results. An MRI study [97] reported poor visual function and severe papilloedema or optic atrophy in association with a larger optic canal, while a CT imaging study [98] found no correlation between any of the optic canal measurements and papilloedema grade and no significant difference in optic canal measurements between IIH patients and controls. 
For the majority, IIH is a chronic condition with disabling headaches that necessitates active management [56]. Migraine headaches are common in IIH patients $[99,100]$. Headache has been shown to be one of the most important determinants of quality of life in individuals with IIH [7]. There appears to be a lack of correlation between headache frequency and severity, and recorded LP opening pressure [100], and the latest guidelines for the classification of headache attributable to IIH have removed reference to improvement of headache with reduction of ICP [58]. The IIH consensus guidelines recommend a stepwise approach for pharmacological treatment of headache [1]. Medication overuse headache is common in IIH and should be eliminated to optimise subsequent therapy [8]. Once the ICP has been reduced, migraine abortive medications may be more effective. Preventive migraine pharmacotherapy can then follow. It is important to avoid medications that have side effects which result in weight gain, due to the strong correlation between weight gain and IIH symptomology $[1,61]$. Drugs that can potentially alter mood should generally be avoided $[1,61]$.

\section{CONCLUSIONS}

IIH is a challenging disease which crosses many medical disciplines. Guidelines are helping clinicians to benchmark their management protocols. With increasing incidence of $\mathrm{IIH}$, there is no better time to explore the underlying pathophysiology to help inform discovery of novel therapeutic agents to improve treatment of this disabling disease.

\section{ACKNOWLEDGEMENTS}

Funding. No funding or sponsorship was received for this study or publication of this article. Alex J. Sinclair is funded by a Sir Jules Thorn Award for Biomedical Science.

Authorship. All named authors meet the International Committee of Medical Journal
Editors (ICMJE) criteria for authorship for this article, take responsibility for the integrity of the work as a whole, and have given their approval for this version to be published.

Disclosures. Susan P. Mollan-Invex Therapeutics advisory board (2019); Heidelberg Engineering speaker fees (2019). Alex J. Sinclair-Invex Therapeutics, company director with salary and stock options $(2019,2020)$. Jasvir Virdee, Stephanie Larcombe, Vivek Vijay and Margaret Dayan do not have a conflict of interest in the subject matter.

Compliance with Ethics Guidelines. This article is based on previously conducted studies and does not contain any studies with human participants or animals performed by any of the authors.

Data Availability. All data generated or analysed during this study are included in this published article/as supplementary information files.

Open Access. This article is licensed under a Creative Commons Attribution-NonCommercial 4.0 International License, which permits any non-commercial use, sharing, adaptation, distribution and reproduction in any medium or format, as long as you give appropriate credit to the original author(s) and the source, provide a link to the Creative Commons licence, and indicate if changes were made. The images or other third party material in this article are included in the article's Creative Commons licence, unless indicated otherwise in a credit line to the material. If material is not included in the article's Creative Commons licence and your intended use is not permitted by statutory regulation or exceeds the permitted use, you will need to obtain permission directly from the copyright holder. To view a copy of this licence, visit http://creativecommons.org/licenses/by$\mathrm{nc} / 4.0 /$. 


\section{REFERENCES}

1. Mollan SP, et al. Idiopathic intracranial hypertension: consensus guidelines on management. J Neurol Neurosurg Psychiatry. 2018;89:1088-100.

2. Markey KA, Mollan SP, Jensen RH, Sinclair AJ. Understanding idiopathic intracranial hypertension: mechanisms, management, and future directions. Lancet Neurol. 2016;15:78-91.

3. Mollan SP, Aguiar M, Evison F, Frew E, Sinclair AJ. The expanding burden of idiopathic intracranial hypertension. Eye (Lond). 2019;33:478-85.

4. Adderley NJ, et al. Association between idiopathic intracranial hypertension and risk of cardiovascular diseases in women in the United Kingdom. JAMA Neurol. 2019;76:1088-98.

5. McCluskey G, et al. Meta-analysis and systematic review of population-based epidemiological studies in idiopathic intracranial hypertension. Eur J Neurol. 2018;25:1218-27.

6. Andrews LE, Liu GT, Ko MW. Idiopathic intracranial hypertension and obesity. Horm Res Paediatr. 2014;81:217-25.

7. Mulla Y, et al. Headache determines quality of life in idiopathic intracranial hypertension. J. Headache Pain. 2015;16:521.

8. Yri HM, Rönnbäck C, Wegener M, Hamann S, Jensen RH. The course of headache in idiopathic intracranial hypertension: a 12-month prospective follow-up study. Eur J Neurol. 2014;21:1458-64.

9. Best J, Silvestri G, Burton B, Foot B, Acheson J. The incidence of blindness due to idiopathic intracranial hypertension in the UK. Open Ophthalmol J. 2013;7:26-9.

10. Ball AK, et al. A randomised controlled trial of treatment for idiopathic intracranial hypertension. J Neurol. 2011;258:874-81.

11. Mollan S, et al. What are the research priorities for idiopathic intracranial hypertension? A priority setting partnership between patients and healthcare professionals. BMJ Open. 2019;9: e026573-e026573026573.

12. Mitchell JL, Mollan SP, Vijay V, Sinclair AJ. Novel advances in monitoring and therapeutic approaches in idiopathic intracranial hypertension. Curr Opin Neurol. 2019;32:422-31.

13. Raoof N, Sharrack B, Pepper IM, Hickman SJ. The incidence and prevalence of idiopathic intracranial hypertension in Sheffield, UK. Eur J Neurol. 2011;18:1266-8.

14. Carta A, et al. Idiopathic intracranial hypertension (Pseudotumor Cerebri): descriptive epidemiology, clinical features, and visual outcome in Parma, Italy, 1990 to 1999. Eur J Ophthalmol. 2004;14: 48-544.

15. Kesler A, Stolovic N, Bluednikov Y, Shohat T. The incidence of idiopathic intracranial hypertension in Israel from 2005 to 2007: results of a nationwide survey. Eur J Neurol. 2014;21:1055-9.

16. Radhakrishnan K, Ahlskog JE, Cross SA, Kurland LT, O'Fallon WM. Idiopathic intracranial hypertension (Pseudotumor Cerebri): descriptive epidemiology in Rochester, Minn, 1976 to 1990. Arch Neurol. 1993;50:78-80.

17. Yabe I, Moriwaka F, Notoya A, Ohtaki M, Tashiro K. Incidence of idiopathic intracranial hypertension in Hokkaido, the northern-most island in Japan. J Neurol. 2000;247:474-5.

18. Liu I-H, Wang A-G, Yen M-Y. Idiopathic intracranial hypertension: clinical features in Chinese patients. Jpn J Ophthalmol. 2011;55:138-42.

19. Tibussek D, Distelmaier F, Kries R, Mayatepek E. Pseudotumor Cerebri in childhood and adolescence-results of a Germany-wide ESPED-survey. Klin Padiatr. 2013;225:81-5.

20. Matthews Y-Y, et al. Pseudotumor cerebri syndrome in childhood: incidence, clinical profile and risk factors in a national prospective population-based cohort study. Arch Dis Child. 2017;102:715-21.

21. Sheldon CA, et al. Pediatric idiopathic intracranial hypertension: age, gender, and anthropometric features at diagnosis in a large, retrospective, multisite cohort. Ophthalmology. 2016;123:2424-31.

22. Mollan SP, et al. Evolving evidence in adult idiopathic intracranial hypertension: pathophysiology and management. J Neurol Neurosurg Psychiatry. 2016;87:982-92.

23. Hornby C, Mollan SP, Botfield H, O'Reilly MW, Sinclair AJ. Metabolic concepts in idiopathic intracranial hypertension and their potential for therapeutic intervention. J Neuroophthalmol. 2018;38:522-30.

24. Ko MW, et al. Weight gain and recurrence in idiopathic intracranial hypertension. Neurology. 2011;76:1564-7.

25. World Health Organisation. Obesity and overweight (2020). https://www.who.int/news-room/ 
fact-sheets/detail/obesity-and-overweight. Accessed 2 Sept 2020

26. Almarzouqi SJ, Morgan M, Lee AG. Idiopathic intracranial hypertension in the Middle East: a growing concern. Saudi J Ophthalmol. 2015;29: 26-31.

27. Daniels AB, et al. Profiles of obesity, weight gain, and quality of life in idiopathic intracranial hypertension (Pseudotumor Cerebri). Am J Ophthalmol. 2007;143:635-641.e1.

28. Newborg B. Pseudotumor Cerebri treated: by ricereduction diet. Arch Intern Med. 1974;133:802-7.

29. Sugerman HJ, Felton WL, Salvant JB, Sismanis A, Kellum JM. Effects of surgically induced weight loss on idiopathic intracranial hypertension in morbid obesity. Neurology. 1995;45:1655-9.

30. Sinclair AJ, et al. Low energy diet and intracranial pressure in women with idiopathic intracranial hypertension: prospective cohort study. BMJ. 2010;341:c2701-c27012701.

31. Committee NIIHSGW, et al. Effect of acetazolamide on visual function in patients with idiopathic intracranial hypertension and mild visual loss: the idiopathic intracranial hypertension treatment trial. JAMA. 2014;311:1641-51.

32. Hornby C, et al. Evaluating the fat distribution in idiopathic intracranial hypertension using dualenergy X-ray absorptiometry scanning. Neuroophthalmology. 2017;42:99-104.

33. Subramaniam S, Fletcher WA. Obesity and weight loss in idiopathic intracranial hypertension: a narrative review. J Neuro-Ophthalmol. 2017;37: 197-205.

34. Speake T, Whitwell C, Kajita H, Majid A, Brown PD. Mechanisms of CSF secretion by the choroid plexus. Microsc Res Tech. 2001;52:49-59.

35. Brown PD, Davies SL, Speake T, Millar ID. Molecular mechanisms of cerebrospinal fluid production. Neuroscience. 2004;129:957-70.

36. Pollay $\mathrm{M}$, et al. Choroid plexus $\mathrm{Na}^{+} / \mathrm{K}^{+}$-activated adenosine triphosphatase and cerebrospinal fluid formation. Neurosurgery. 1985;17:768-72.

37. Kerty E, et al. Is the brain water channel aquaporin4 a pathogenetic factor in idiopathic intracranial hypertension? Results from a combined clinical and genetic study in a Norwegian cohort. Acta Ophthalmol. 2013;91:88-91.
38. Stiebel-Kalish H, Eyal S, Steiner I. The role of aquaporin-1 in idiopathic and drug-induced intracranial hypertension. Med Hypotheses. 2013;81:1059-62.

39. Uldall $\mathrm{M}$, et al. Choroid plexus aquaporin 1 and intracranial pressure are increased in obese rats: towards an idiopathic intracranial hypertension model? Int J Obes. 2017;41:1141-7.

40. Killer $\mathrm{HE}$, et al. Cerebrospinal fluid dynamics between the basal cisterns and the subarachnoid space of the optic nerve in patients with papilloedema. Br J Ophthalmol. 2011;95:822-7.

41. Sussman J, Leach M, Greaves M, Malia R, DaviesJones GA. Potentially prothrombotic abnormalities of coagulation in benign intracranial hypertension. J Neurol Neurosurg Psychiatry. 1997;62:229-33.

42. Botfield HF, et al. A glucagon-like peptide-1 receptor agonist reduces intracranial pressure in a rat model of hydrocephalus. Sci Transl Med. 2017;9:eaan0972.

43. Sinclair AJ, et al. Corticosteroids, 11ß-hydroxysteroid dehydrogenase isozymes and the rabbit choroid plexus. J Neuroendocrinol. 2007;19: 614-20.

44. Sinclair AJ, et al. Cerebrospinal fluid corticosteroid levels and cortisol metabolism in patients with idiopathic intracranial hypertension: a link between $11 \beta-H S D 1$ and intracranial pressure regulation? J Clin Endocrinol Metab. 2010;95:5348-56.

45. Markey KA, et al. Assessing the efficacy and safety of an $11 \beta$-hydroxysteroid dehydrogenase type 1 inhibitor (AZD4017) in the idiopathic intracranial hypertension drug trial, IIH:DT: clinical methods and design for a phase II randomized controlled trial. JMIR Res Protoc. 2017;6:e181-e181181.

46. Hornby C, et al. What do transgender patients teach us about idiopathic intracranial hypertension? Neuro-Ophthalmol. 2017;41:326-9.

47. O'Reilly MW, et al. A unique androgen excess signature in idiopathic intracranial hypertension is linked to cerebrospinal fluid dynamics. JCI Insight. 2019;4:e125348.

48. Zhang Y, Scarpace PJ. The role of leptin in leptin resistance and obesity. Physiol Behav. 2006;88: 249-56.

49. Lampl Y, et al. Serum leptin level in women with idiopathic intracranial hypertension. J Neurol Neurosurg Psychiatry. 2002;72:642-3.

50. Ball AK, et al. Elevated cerebrospinal fluid (CSF) leptin in idiopathic intracranial hypertension (IIH): evidence for hypothalamic leptin resistance? Clin Endocrinol (Oxf). 2009;70:863-9. 
51. Van Harmelen V, et al. Leptin secretion from subcutaneous and visceral adipose tissue in women. Diabetes. 1998;47:913-7.

52. Riggeal BD, et al. Clinical course of idiopathic intracranial hypertension with transverse sinus stenosis. Neurology. 2013;80:289-95.

53. Bidot $\mathrm{S}$, et al. Brain Imaging in Idiopathic Intracranial Hypertension. J. Neuro-Ophthalmol. 2015;35: 400-11.

54. King JO, Mitchell PJ, Thomson KR, Tress BM. Manometry combined with cervical puncture in idiopathic intracranial hypertension. Neurology. 2002;58:26-30.

55. Saber H, Lewis W, Sadeghi M, Rajah G, Narayanan S. Stent survival and stent-adjacent stenosis rates following venous sinus stenting for idiopathic intracranial hypertension: a systematic review and meta-analysis. Interv Neurol. 2018;7:490-500.

56. Mollan SP, Spitzer D, Nicholl DJ. Raised intracranial pressure in those presenting with headache. BMJ. 2018;363:1-5.

57. Mollan SP, Hoffmann J, Sinclair AJ. Advances in the understanding of headache in idiopathic intracranial hypertension. Curr Opin Neurol. 2019;32:92-8.

58. Vincent M, Wang S. Headache Classification Committee of the International Headache Society (IHS) The International Classification of Headache Disorders, 3rd edition. Cephalalgia. 2018;38:1-211.

59. Yri HM, Fagerlund B, Forchhammer HB, Jensen RH. Cognitive function in idiopathic intracranial hypertension: a prospective case-control study. BMJ Open. 2014;4:e004376-e00437604376.

60. Fisayo A, Bruce BB, Newman NJ, Biousse V. Overdiagnosis of idiopathic intracranial hypertension. Neurology. 2016;86:341-50.

61. Hoffmann J, et al. European headache federation guideline on idiopathic intracranial hypertension. J Headache Pain. 2018;19:93.

62. Blanch RJ, et al. Detection of Papilloedema Study (DOPS): rates of false positive papilloedema in the community. Eye. 2019;33:1073-80.

63. Fard MA, et al. Optical coherence tomography angiography in papilledema compared with pseudopapilledema. Investig Ophthalmol Vis Sci. 2019;60:168-75.

64. Malhotra K, Padungkiatsagul T, Moss HE. Optical coherence tomography use in idiopathic intracranial hypertension. Ann Eye Sci. 2020;5:7.
65. Mollan SP, Hornby C, Mitchell J, Sinclair AJ. Evaluation and management of adult idiopathic intracranial hypertension. Pract Neurol. 2018;18: $485-8$.

66. Biousse V, Ameri A, Bousser M-G. Isolated intracranial hypertension as the only sign of cerebral venous thrombosis. Neurology. 1999;53: 1537-1537.

67. Friedman DI, Liu GT, Digre KB. Revised diagnostic criteria for the pseudotumor cerebri syndrome in adults and children. Neurology. 2013;81:1159-65.

68. Mollan SP, et al. Idiopathic intracranial hypertension associated with iron deficiency anaemia: a lesson for management. Eur Neurol. 2009;62:105-8.

69. Benzimra JD, Simon S, Sinclair AJ, Mollan SP. Sightthreatening pseudotumour cerebri associated with excess vitamin A supplementation. Pract Neurol. $2015 ; 15: 72-3$.

70. Mollan SP, et al. A practical approach to, diagnosis, assessment and management of idiopathic intracranial hypertension. Pract Neurol. 2014;14: 380-90.

71. Kalyvas A, et al. A systematic review of surgical treatments of idiopathic intracranial hypertension (IIH). Neurosurg Rev. 2020. https://doi.org/10. 1007/s10143-020-01288-1.

72. Sinclair AJ, et al. Is cerebrospinal fluid shunting in idiopathic intracranial hypertension worthwhile? A 10-year review. Cephalalgia. 2011;31:1627-33.

73. Kalyvas AV, et al. Efficacy, complications and cost of surgical interventions for idiopathic intracranial hypertension: a systematic review of the literature. Acta Neurochir (Wien). 2017;159:33-49.

74. Gilbert AL, Chwalisz B, Mallery R. Complications of optic nerve sheath fenestration as a treatment for idiopathic intracranial hypertension. Semin Ophthalmol. 2018;33:36-41.

75. Ross Middleton KM, Patidar SM, Perri MG. The impact of extended care on the long-term maintenance of weight loss: a systematic review and metaanalysis. Obes Rev. 2012;13:509-17.

76. Sjöström L. Review of the key results from the Swedish Obese Subjects (SOS) trial-a prospective controlled intervention study of bariatric surgery. J Intern Med. 2013;273:219-34.

77. Rubino F, et al. The early effect of the Roux-en-Y gastric bypass on hormones involved in body weight regulation and glucose metabolism. Ann Surg. 2004;240:236-42. 
78. Abdelbaki TN, Gomaa M. Outcome of idiopathic intracranial hypertension after laparoscopic sleeve gastrectomy. Surg Obes Relat Dis. 2020. https://doi. org/10.1016/j.soard.2020.03.032.

79. Ottridge R, et al. Randomised controlled trial of bariatric surgery versus a community weight loss programme for the sustained treatment of idiopathic intracranial hypertension: the Idiopathic Intracranial Hypertension Weight Trial (IIH:WT) protocol. BMJ Open. 2017;7: e017426-e017426017426.

80. Piper RJ, et al. Interventions for idiopathic intracranial hypertension. Cochrane database Syst Rev. 2015;2015:CD003434.

81. Çelebisoy N, Gökçay F, Şirin H, Akyürekli Ö. Treatment of idiopathic intracranial hypertension: topiramate vs acetazolamide, an open-label study. Acta Neurol Scand. 2007;116:322-7.

82. Scotton WJ, et al. Topiramate is more effective than acetazolamide at lowering intracranial pressure. Cephalalgia. 2019;39:209-18.

83. Markey K, et al. 11 $\beta$-Hydroxysteroid dehydrogenase type 1 inhibition in idiopathic intracranial hypertension: a double-blind randomized controlled trial. Brain Commun. 2020;2:2.

84. Yiangou A, et al. Therapeutic lumbar puncture for headache in idiopathic intracranial hypertension: minimal gain, is it worth the pain? Cephalalgia. 2019;39:245-53.

85. Scotton WJ, et al. Characterising the patient experience of diagnostic lumbar puncture in idiopathic intracranial hypertension: a cross-sectional online survey. BMJ Open. 2018;8: e020445-e020445020445.

86. Mollan SP, Mitchell JL, Sinclair AJ. Tip of the iceberg in idiopathic intracranial hypertension. Pract Neurol. 2019;19:178-9.

87. Liu KC, et al. Venous sinus stenting for reduction of intracranial pressure in IIH: a prospective pilot study. J Neurosurg. 2017;127:1126-33.

88. Matloob SA, et al. Effect of venous stenting on intracranial pressure in idiopathic intracranial hypertension. Acta Neurochir (Wien). 2017;159: 1429-37.

89. Puffer RC, Mustafa W, Lanzino G. Venous sinus stenting for idiopathic intracranial hypertension: a review of the literature. J Neurointerv Surg. 2013;5: 483-6.

90. Nicholson P, et al. Venous sinus stenting for idiopathic intracranial hypertension: a systematic review and meta-analysis. J Neurointerv Surg. 2019;11:380-5.

91. Dinkin MJ, Patsalides A. Venous sinus stenting for idiopathic intracranial hypertension: where are we now? Neurol Clin. 2017;35:59-81.

92. Gurney SP, Ramalingam S, Thomas A, Sinclair AJ, Mollan SP. Exploring the current management idiopathic intracranial hypertension, and understanding the role of dural venous sinus stenting. Eye Brain. 2020;12:1-13.

93. Marzoli SB, et al. Quantitative analysis of optic nerve damage in idiopathic intracranial hypertension (IIH) at diagnosis. Neurol Sci. 2013;34:143-5.

94. Aojula A, et al. Segmentation error in spectral domain optical coherence tomography measures of the retinal nerve fibre layer thickness in idiopathic intracranial hypertension. BMC Ophthalmol. 2018;17:257.

95. Micieli JA, et al. Optic nerve appearance as a predictor of visual outcome in patients with idiopathic intracranial hypertension. $\mathrm{Br} \mathrm{J}$ Ophthalmol. 2019;103:1429-35.

96. Blanch RJ, et al. Differing presenting features of idiopathic intracranial hypertension in the UK and US. Eye. 2019;33:1014-9.

97. Bidot $S$, et al. The optic canal size is associated with the severity of papilledema and poor visual function in idiopathic intracranial hypertension. J Neuro-Ophthalmol. 2016;36:120-5.

98. Skipper N, et al. Do optic canal dimensions measured on CT influence the degree of papiloledema and visual dysfunction in idiopathic intracranial hypertension? Neuro-Ophthalmology. 2018;43:1-7.

99. Sina F, Razmeh S, Habibzadeh N, Zavari A, Nabovvati $M$. Migraine headache in patients with idiopathic intracranial hypertension. Neurol Int. 2017;9:7280.

100. Friedman DI, et al. Headache in idiopathic intracranial hypertension: findings from the idiopathic intracranial hypertension treatment trial. Headache J Head Face Pain. 2017;57:1195-205. 